\title{
Structural Analysis And Topology Optimization Design For Bandwidth Extension of Magnetoelectric Seismometer
}

\author{
Zhenjing Yao ( $\nabla$ yaozhenjing000@126.com ) \\ Institute of Disaster Prevention \\ Jingyi Zhang \\ Institute of Disaster Prevention \\ Zhitao Gao \\ Institute of Disaster Prevention \\ Yaran Liu \\ Institute of Disaster Prevention \\ Mingyang Li \\ Institute of Disaster Prevention
}

\section{Research Article}

Keywords: Seismometer, Frequency bandwidth, Natural frequency, Spurious frequency, Topology optimization

Posted Date: November 18th, 2021

DOI: https://doi.org/10.21203/rs.3.rs-1037972/v1

License: (c) (i) This work is licensed under a Creative Commons Attribution 4.0 International License. Read Full License 


\section{Structural analysis and topology optimization design for bandwidth}

\section{2 extension of magnetoelectric seismometer}

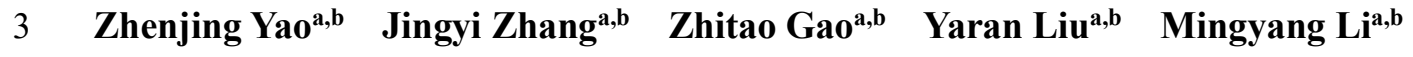

4 a Institute of Disaster Prevention, Sanhe 065201, China

5 b Hebei Key Laboratory of Seismic Disaster Instrument and Monitoring Technology, Sanhe 065201, China

\section{ABSTRACT}

Magnetoelectric seismometers can measure earthquake information and play an important role in earthquake monitoring. Aiming at the wider effective frequency bandwidth of magnetoelectric seismometers, a novel seismometer based on topology optimization structural pendulum is reported. The topology optimization of leaf spring structure in magnetoelectric seismometer is designed, the natural frequency and spurious frequency characteristics of the novel seismometer are analyzed. Based on variable density theory, the Solid Isotropic Material with Penalization (SIMP) model of the seismometer is established, and the Method of Moving Asymmetric (MMA) is adopted to obtain the optimal topology structure. The finite element analysis using ANSYS shows that novel seismometer after topology optimization structure is characteristic with lower natural frequency and higher spurious frequency than that of before optimization seismometer. The real vibration experimental results indicate that after topology optimization, the effective frequency bandwidth of seismometer is increased by $55.50 \%$, improving from $[1 \mathrm{~s}, 51 \mathrm{~Hz}]$ to $[4 \mathrm{~s}, 78 \mathrm{~Hz}]$.

Keywords: Seismometer, Frequency bandwidth, Natural frequency, Spurious frequency, Topology optimization.

\section{Introduction}

Seismometers, as ground motion measurement instruments, have been widely used in geological hazard predictions, earthquake early warning, engineering exploration and nuclear explosions monitoring [1-4]. A seismometer can turn ground velocity within a certain bandwidth into an electric output signal without distortion [5]. The response signal of seismometer will decline or become distorted when it falls below natural frequency or near spurious frequency. Therefore, seismometers with a lower natural frequency and a higher spurious frequency play an important role in ground motion measurements with wider bandwidth, ensuring the reliability of quality data acquisition.

The structural changes in leaf spring of seismometer can search for lower natural frequency and higher spurious frequency with wider effective frequency bandwidth to a certain extent. Faber and Maxwell alter the leaf spring structure of a seismometer to increase spurious frequency which can expand its frequency bandwidth [6]. Woo invents inner and outer annular rings connected by spring arm for a seismometer, which improves the ratio between spurious frequency and natural frequency to broaden bandwidth and reduce signal distortion [7]. Wielandt and Streckeisen propose a seismometer with a rectangular leaf spring instead of a zero-length helical spring, which has a broader bandwidth [8]. 
spurious frequency to natural frequency in order to expand its frequency range [9]. Xin et al. design a multilayer spiral-corrugated cantilever beam for a piezoelectric seismometer, which has a lower natural frequency [10]. Yao et al. propose a novel leaf spring as a substitute for traditional spring to expand effective bandwidth of a seismometer [11]. The existing topology structure of original leaf spring can search for lower natural frequency and higher spurious frequency in a certain extent. However, the topology structure of leaf spring cannot be optimized which has a large space for expanding bandwidth of a seismometer.

This paper demonstrates how the novel seismometer with topology optimization leaf spring structure can improve the effective frequency bandwidth. The proposed topology optimization leaf spring structure with lower natural frequency and higher spurious frequency can broaden frequency bandwidth. Based on variable density theory, the Solid Isotropic Material with Penalization (SIMP) model of seismometer is established, and the Method of Moving Asymmetric (MMA) is adopted to obtain the optimal topology structure. As well as the natural frequency and spurious frequency characteristics of novel topology optimization structure seismometer are analyzed. The finite element simulation analysis using ANSYS Workbench 19.0 and real measurement experiments are conducted to confirm validity of the design.

The rest of this paper is structured as follows: The working principles are explained in Sec. 2; Sec. 3 presents structural analysis; Topology optimization and design of novel topology seismometer is introduced in Sec. 4 and Sec. 5; Sec. 6 illustrates finite element simulation analysis; Sec. 7 represents experiments and discussions, and conclusions in Sec.8.

\section{Working principles}

The ground motion measurement can directly sense by the mechanical pendulum of seismometer [12]. It suspends an inertial reference mass structure from a rigid fixed shell structure, which is connected by a leaf spring. When local seismic waves occur, the fixed shell structure coupled with the ground vibrates immediately. The inertial reference mass still tends to remain static, so the relative movement is generated between mass and shell. Using this relative motion, the mass-spring-damping mechanical model. 


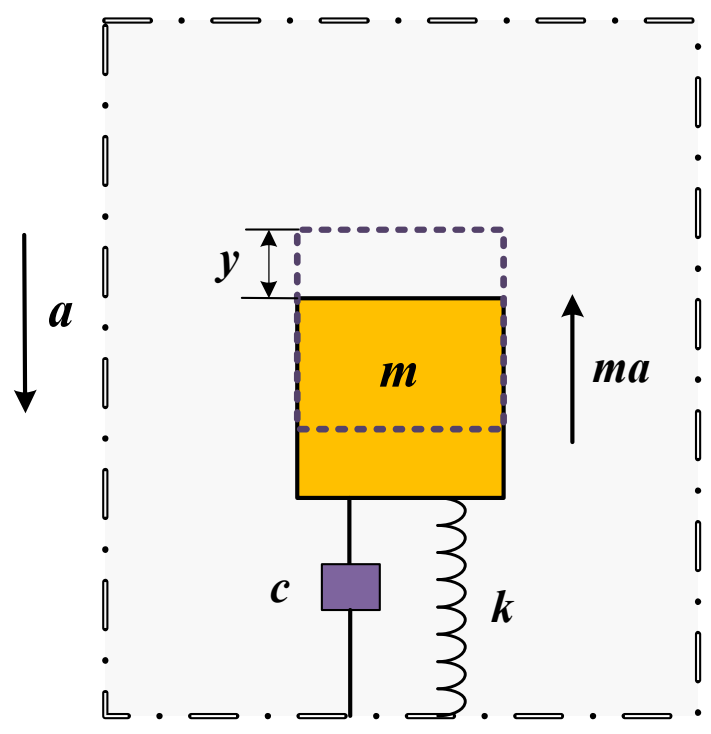

Fig. 1. Model of the mechanical pendulum

Under the ideal conditions, when the displacement of ground motion $Y$ produces relative displacement $y$ between the mass and shell, the motion equation of mechanical pendulum can be shown as

$$
m \frac{\mathrm{d}^{2} y}{\mathrm{~d} t^{2}}+c \frac{\mathrm{d} y}{\mathrm{~d} t}+k y=m \frac{\mathrm{d}^{2} Y}{\mathrm{~d} t^{2}}
$$

Wherein, $m$ represents the quality of inertial reference mass structure, $k$ is the stiffness of connecting leaf spring, and $c$ represents the damping coefficient of mechanical pendulum.

Assuming that damping can be neglected, the mechanical pendulum vibrates in simple harmonic. With finite element discretization of the mechanical pendulum vibration motion, after element analysis and boundary condition, the motion equation with finite element of mechanical pendulum can be represented as

$$
\mathbf{M y}+\mathbf{K y}=\mathbf{0}
$$

Where $\mathbf{M}$ and $\mathbf{K}$ are the mass matrix and stiffness matrix of mechanical pendulum, respectively. $\mathbf{y}$ and $\ddot{\mathbf{y}}$ are the displacement vector and acceleration vector of mechanical pendulum, respectively.

When the mechanical pendulum vibrates in simple harmonic, the natural vibration frequency equation of the mechanical pendulum can be shown as follows,

$$
\left(\mathbf{K}-\omega^{2} \mathbf{M}\right) \boldsymbol{\Phi}=\mathbf{0}
$$

Where $\omega$ represents the vibration angular frequency of mechanical pendulum, and feature vector $\boldsymbol{\Phi}$ is the vibration mode of mechanical pendulum.

In this way, the $j$-order frequency $f_{j}$ of mechanical pendulum can be expressed as 


$$
f_{j}=\frac{1}{2 \pi} \sqrt{\frac{\boldsymbol{\Phi}_{j}^{\mathrm{T}} \mathbf{K} \boldsymbol{\Phi}_{j}}{\boldsymbol{\Phi}_{j}^{\mathrm{T}} \mathbf{M} \boldsymbol{\Phi}_{j}}}
$$

Where $\boldsymbol{\Phi}_{j}$ is feature vector related to the $j$-order angular frequency of mechanical pendulum. As can be seen from equation (4), the $j$-order frequency of mechanical pendulum depends only on the stiffness matrix $\mathbf{K}$ and the mass matrix M. Order to minimize natural frequency and maximize spurious frequency of mechanical pendulum, structural analysis and design of topology optimization leaf spring in rectangle structure is considered, which can change the stiffness matrix and mass matrix of mechanical pendulum.

\section{Structural analysis}

The working principle of magnetoelectric seismometer is that the mechanical pendulum directly perceives the ground vibration, and the connected working coil cuts the magnetic induction line in the magnetic field, then converts the ground motion into electricity for measurement. The key structure of mechanical pendulum is composed of leaf spring, counterweight, beam, heavy hammer, coil support, large coil frame and pressure plate as shown in Fig 2.

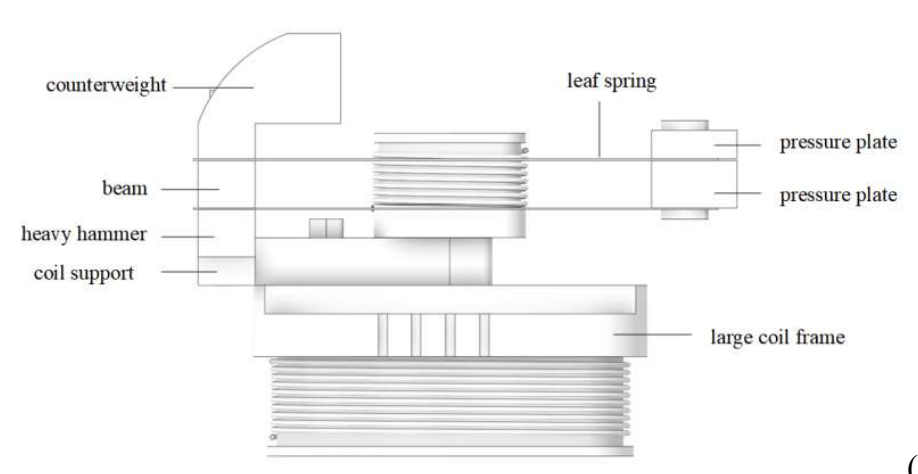

(a)

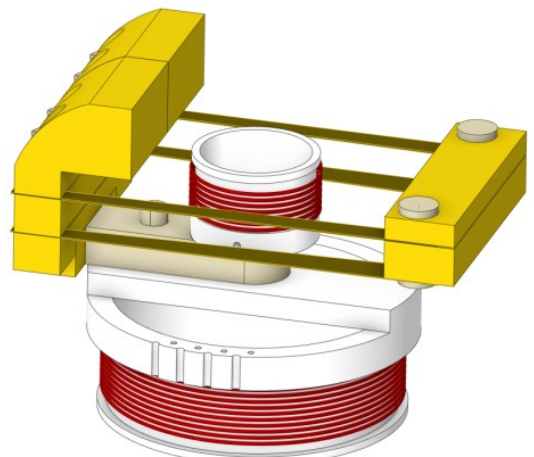

(b)

Fig. 2. (a) Struture of mechanical pendulum and (b) 3D diagram of mechanical pendulum in magnetoelectric seismometer

The finite element analysis model of mechanical pendulum is built to study the influence of leaf spring size on the natural frequency and spurious frequency. After solid modeling, defining material property and meshing grid, the unit and node number in finite element model is set to 84349 and 148061 , respectively. When seismometer measures the ground vibration, the hammer end connected by the leaf spring swing freely and the pressure plate end connected by the leaf spring is fixed. So it has fixed constraints on the end of pressure plate. Loading the stand earth gravity, the finite element analysis of mechanical pendulum is carried out. The first order modal shape and the second order modal shape of mechanical pendulum is shown in Figure 3. It can be seen from Fig. 3(a) that the main vibration of the first order modal of mechanical pendulum is bending motion, while the main vibration of the second order modal of mechanical pendulum is twisting motion in Fig. 3(b). In the seismometer measurement, the actual motion of the mechanical 
pendulum is the up and down variation, while the twisting motion is the unexpected motion. Moreover, the excessive twisting motion could destroy the mechanical pendulum, so it is necessary to has a lower natural frequency and higher spurious frequency of the mechanical pendulum.
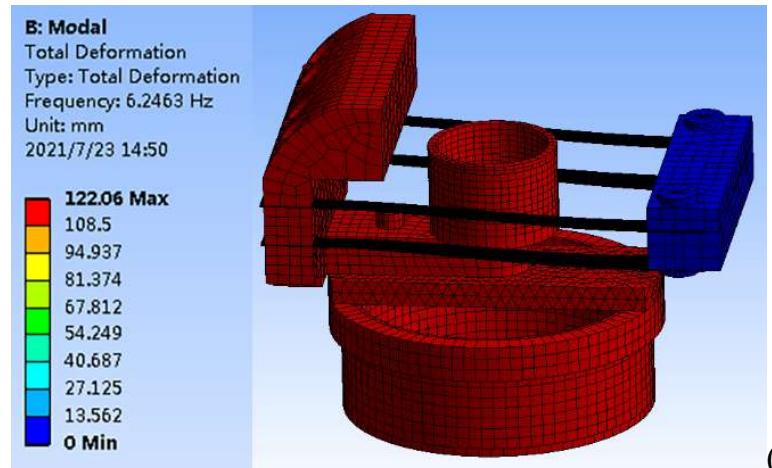

(a)
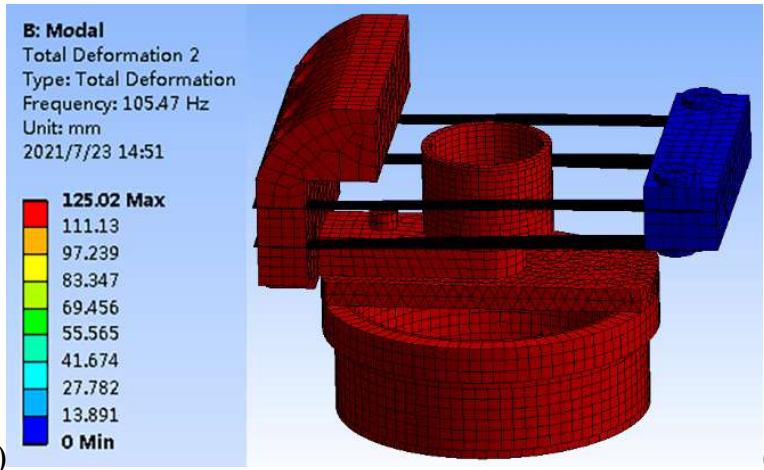

(b)

Fig. 3. The finite element analysis (a) the first order modal shape (b) the second order modal shape

The size of leaf spring is a key factor affecting the natural frequency and spurious frequency of mechanical pendulum. In order to obtain the variation laws between the size and frequency of mechanical pendulum, changing the length, width and thickness of leaf spring, the corresponding natural frequency and spurious frequency of the mechanical pendulum are analyzed.

In finite element model of the mechanical pendulum, the length of the leaf spring is $30.00-85.00 \mathrm{~mm}$ at $5.00 \mathrm{~mm}$ interval, the width of the leaf spring is $0-5.50 \mathrm{~mm}$ at $0.50 \mathrm{~mm}$ interval, and the thickness of the leaf spring is $0-0.55 \mathrm{~mm}$ at $0.05 \mathrm{~mm}$ interval. With the increase of the leaf spring length, the natural frequency of the pendulum decreases from $18.71 \mathrm{~Hz}$ to $3.04 \mathrm{~Hz}$, and the spurious frequency decreases from $85.49 \mathrm{~Hz}$ to $16.04 \mathrm{~Hz}$, and the difference between the natural frequency and the spurious frequency of the mechanical pendulum decreases from $66.78 \mathrm{~Hz}$ to $12.99 \mathrm{~Hz}$ as shown in Fig 4(a). With the increase of the spring width, the natural frequency increases from $2.31 \mathrm{~Hz}$ to $7.14 \mathrm{~Hz}$, the spurious frequency increases from $3.60 \mathrm{~Hz}$ to $43.31 \mathrm{~Hz}$, and the difference between the natural frequency and the spurious frequency of the pendulum increases from $1.29 \mathrm{~Hz}$ to $36.17 \mathrm{~Hz}$ as shown in Fig $4(\mathrm{~b})$. With the thickness of the leaf spring increases from 0 to $0.55 \mathrm{~mm}$, the natural frequency of the pendulum increases from $3.49 \mathrm{~Hz}$ to $50.63 \mathrm{~Hz}$ and the spurious frequency from $25.42 \mathrm{~Hz}$ to $85.98 \mathrm{~Hz}$, and the difference between the natural frequency and the spurious frequency of the pendulum increases from $21.93 \mathrm{~Hz}$ to $35.35 \mathrm{~Hz}$ as shown in Fig 4(c). 


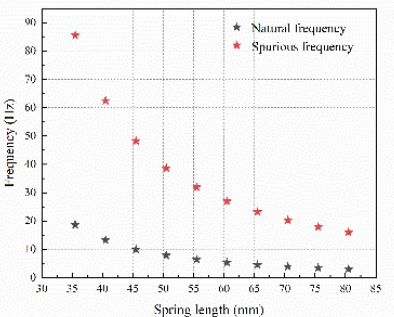

(a)

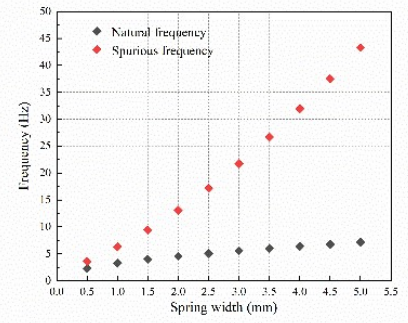

(b)

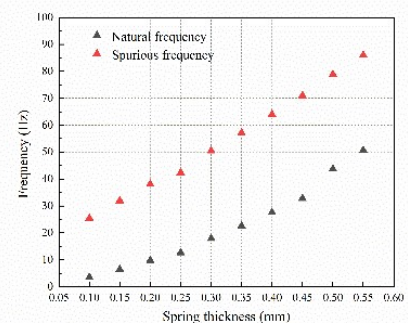

(c)

119 Fig. 4. The variation laws between the size and frequency of mechanical pendulum: (a) change in natural frequency 120 and spurious frequency with spring length (b) change in natural frequency and spurious frequency with spring width (c) change in natural frequency and spurious frequency with spring thickness.

The increase in length of the leaf spring causes a rapid decrease in the difference between natural frequency and spurious frequency of the pendulum, while the increase in width of the leaf spring induces a rapid growth in the difference between natural frequency and spurious frequency of the pendulum, but the increase in thickness of the leaf spring has little effect on the difference between natural frequency and spurious frequency of the pendulum. In this study, the length of the leaf spring is set to $57.50 \mathrm{~mm}$, the width is $4.00 \mathrm{~mm}$, and the thickness is $0.15 \mathrm{~mm}$. The analysis result of the natural frequency is $6.24 \mathrm{~Hz}$, and the spurious frequency is $105.47 \mathrm{~Hz}$.

\section{Topology optimization}

In order to design seismometers with lower natural frequency and higher spurious frequency, a topology optimitial structure of leaf spring is proposed. Topology optimization is to find the optimal distribution of materials in given area and boundary conditions $[13,14]$. Based on variable density theory, the Solid Isotropic Material with Penalization (SIMP) model of seismometer is established, and the problem of minimizing natural frequency of mechanical pendulum can be formulated as follows,

$$
\begin{array}{cl}
\text { Find : } & x=\left(x_{1}, x_{2}, \ldots, x_{N}\right)^{\mathrm{T}} \\
\min : & f=\frac{1}{2 \pi} \sqrt{\frac{\boldsymbol{\Phi}_{1}^{\mathrm{T}} \mathbf{K} \boldsymbol{\Phi}_{1}}{\boldsymbol{\Phi}_{1}^{\mathrm{T}} \mathbf{M} \boldsymbol{\Phi}_{1}}} \\
\text { s.t. } & (\mathbf{K}-\lambda \mathbf{M}) \boldsymbol{\Phi}=\mathbf{0} \\
& \lambda=\mathrm{n} f^{2} \\
& \sum_{i=1}^{N} V_{i} x_{i}-V^{*} \leq 0 \\
& 0 \leq x_{\min } \leq x_{i} \leq 1
\end{array}
$$


Wherein $x$ is the design variable, $x_{i}$ is the unit density, and $x_{\min }$ is the lower limit of the unit density. $N$ represents the element number, $V^{*}$ is the allowable volume of material, $\lambda$ represents eigenvalue, and $f$ is the natural frequency of mechanical pendulum.

The sensitivity of natural frequency in mechanical pendulum can be shown as follows,

$$
\begin{gathered}
\frac{\partial f}{\partial x_{i}}=\boldsymbol{\Phi}_{1}^{\mathrm{T}}\left(\frac{\partial \mathbf{K}}{\partial x_{i}}-f \frac{\partial \mathbf{M}}{\partial x_{i}}\right) \boldsymbol{\Phi}_{1} \\
\mathbf{K}=\sum_{i=1}^{N}\left(E_{\text {min }}+x_{i}^{P}\left(E_{0}-E_{\text {min }}\right)\right) \mathbf{K}_{\mathbf{i}} \\
\mathbf{M}=\sum_{i=1}^{N}\left(E_{\text {min }}+x_{i}^{P}\left(E_{0}-E_{\text {min }}\right)\right) \mathbf{M}_{\mathbf{i}}
\end{gathered}
$$

Where $P$ is the penalty factor for intermediate density materials, $E_{0}$ and $E_{\min }$ are the elastic modulus of materials in material region and hole region, respectively. $\mathbf{K}_{i}$ and $\mathbf{M}_{i}$ are the stiffness and mass of $i$-th element, respectively.

The Method of Moving Asymptote (MMA) is adopted to solve topology optimization problem with SIMP model of seismometer. MMA is a convex approximate linearization method based on the first derivative [15-20]. To improve algorithm performance, the artificial variables are introduced into the SIMP optimization model. The mathematical model can be expressed as follows,

$$
\begin{aligned}
\min : & \frac{1}{2 \pi} \sqrt{\frac{\boldsymbol{\Phi}_{1}^{\mathrm{T}} \mathbf{K \Phi}}{\boldsymbol{\Phi}_{1}^{\mathrm{T}} \mathbf{M \Phi}}}+a_{0} z+\sum_{j=1}^{m}\left(c_{j} y_{j}+\frac{1}{2} d_{j} y_{j}^{2}\right) \\
\text { s.t.: } & f_{j}(x)-a_{j} z-y_{j} \leq 0 \quad j=1,2, \cdots, m \\
& x_{i}^{\min } \leq x_{i} \leq x_{i}^{\max } \quad i=1,2, \cdots, N \\
& z \geq 0, \quad y_{j} \geq 0 \quad j=1,2, \cdots, m
\end{aligned}
$$

Where $y$ and $z$ are artificial variables, $a_{0}, c_{j}, d_{j} a_{j}, x_{i}^{\min }$ and $x_{i}^{\max }$ are real number greater than zero, $m$ is number of constraints.

The steps of MMA to solve seismometer topology optimization problem are that:

Step 1: Selecting the initial iteration point of design variables. The size of initial finite element in design domain is 9562 .

Step 2: Calculating values of the natural frequency function and constraint function, and sensitivity of the natural frequency in mechanical pendulum at the current iteration point of design variable.

Step 3: Solving next iteration point of design variables. Generating MMA sub-problem with adding artificial variables, original dual method is used to solve the approximate solution as the next iteration point of design variables. 
Step 4: Judging the termination conditions. If the convergence condition is met, acquiring the optimal topology structure; if not, return to Step 2.

\section{Design of novel topology optimization seismometer}

Aiming to have a wider effective frequency bandwidth seismometer, we have designed novel seismometer using an optimal topology leaf spring structure pendulum based on SIMP model and MMA algorithm. The design area of mechanical pendulum is the blue aera as shown in Fig. 5(a). For MMA optimization algorithm, the penalty factor is taken to 3 , the convergence accuracy is $0.1 \%$. The main frequency of the central processing unit and random access memory used for implementation of optimization algorithm are $2.6 \mathrm{~Hz}$ and $64 \mathrm{~GB}$, respectively. With the size of initial algorithm, the topology optimization structure leaf spring is as shown in Fig. 5(b). Adopting the topology optimization structure leaf spring, the novel optimization pendulum for a seismometer is assembled. Figures $5(\mathrm{c})-5(\mathrm{~d})$ illustrate the exploded view of the novel optimization pendulum and the finite element model of the novel optimization pendulum,

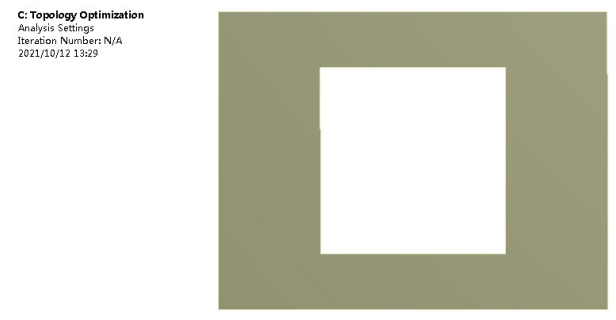

175 (c)

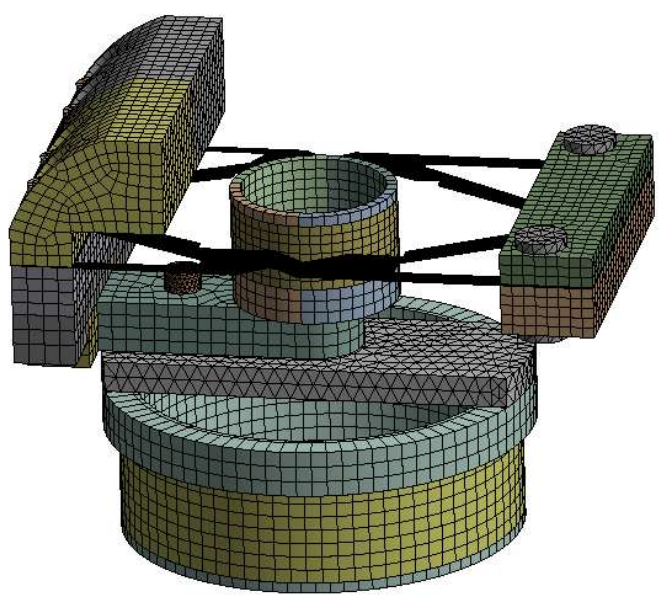

(d)

Fig. 5. Design of the novel seismometer with topology optimization (a) design area of mechanical pendulum; (b) topology optimization leaf spring structure; (c) exploded view of the novel topology pendulum is as follows: (1) counterweight, (2) 
bolts, (3) beam, (4) heavy hammer, (5) coil support, (6) large coil frame, (7) pressure plate, (8) novel optimization leaf spring, (9) pressure plate, (10) small coil frame; (d) finite element model of the novel optimization pendulum.

\section{Finite element simulation analysis}

In order to compare the performance of novel topology optimization leaf spring structure and before optimization leaf spring structure for a seismometer, a finite element analysis using ANSYS Workbench 19.0 (finite element computer program) is conducted. The finite element simulation experiments are carried out by a workstation. The main frequency of central processing unit and the random access memory used for simulation are $2.6 \mathrm{~Hz}$ and $64 \mathrm{~GB}$, respectively. The modal analysis and harmonic response analysis are conducted on two pendulum structures. The natural frequency and spurious frequency of pendulum structure for a seismometer are analyzed.

6.1 Modal analysis

In modal analysis, the natural frequency and spurious frequency of pendulum structure for a seismometer are analyzed under free vibration. The first and second order modal shapes of novel topology optimization pendulum using modal analysis under ANSYS Workbench are carried out. Figure 6(a) present the first order modal shape of novel topology optimization pendulum. Figure 6(b) illustrate the second order modal shape of novel topology optimization pendulum.

From Fig. 6, we can observe that the direction of first order modal shape is concurrent with ground movement, but the direction of second order modal shape is intersected with the ground variation, which can produce a spurious signal for a seismometer. The natural frequency of novel pendulum with topology optimization leaf spring is $3.21 \mathrm{~Hz}$, and the spurious frequency is $124.14 \mathrm{~Hz}$.

B: Modal
Total Deformation
Type: Total Deformation
Frequency: 3.211 Hz
Unit: mm
2021/7/23 14:56
\[ \begin{array}{l}116.48 \text { Max } \\ 103.54 \\ 90.598 \\ 77.655 \\ 64.713 \\ 51.77 \\ 38.828 \\ 25.885 \\ 12.943 \\ 0 \text { Min }\end{array} \]
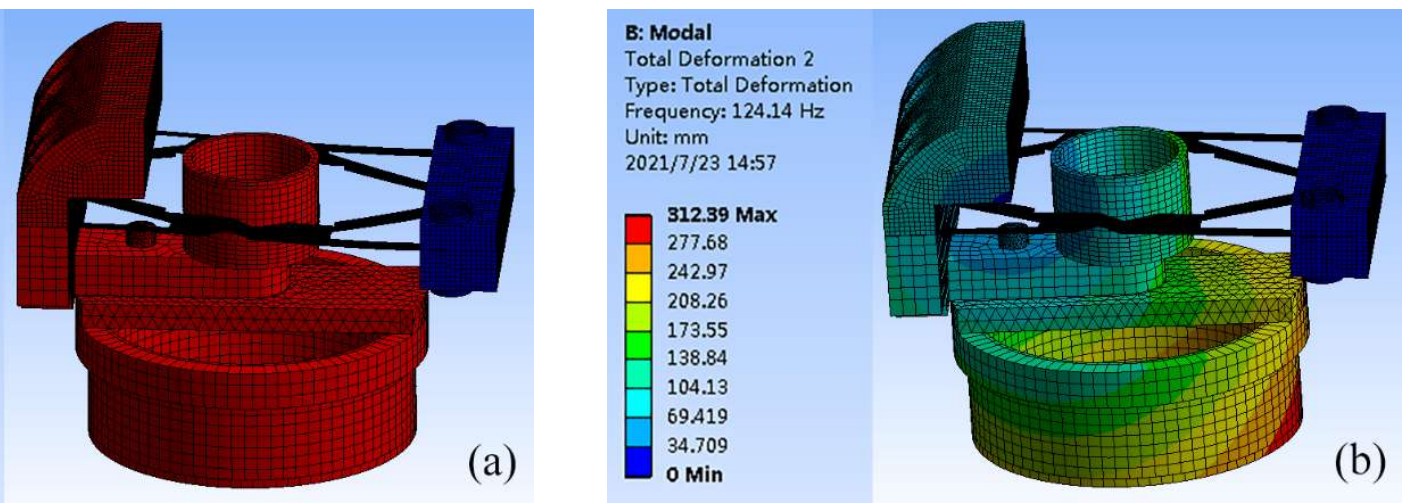

Fig. 6. Modal analysis results (a) The first order modal shape of the novel optimization optimization pendulum, (b) the second order modal shape of the novel optimization pendulum.

Table 1 shows the natural frequency and spurious frequency of two kinds of pendulum. The natural frequency of novel pendulum with topology optimization leaf spring is decreased with before optimization pendulum from $6.24 \mathrm{~Hz}$ to $3.21 \mathrm{~Hz}$, which is $48.55 \%$ reduction. Meanwhile, the spurious frequency of novel optimization pendulum is increased 
203 with that of before optimization pendulum from $105.47 \mathrm{~Hz}$ to $124.14 \mathrm{~Hz}$, which is $17.70 \%$ increase. The finite element 204 analysis shows that novel seismometer with topology optimization structure is characteristic with lower natural frequency 205 and higher spurious frequency than that of before optimization seismometer.

Table 1 Natural frequency and spurious frequency of two kinds of pendulum.

\begin{tabular}{ccc}
\hline & Natural frequency $(\mathrm{Hz})$ & Spurious frequency $(\mathrm{Hz})$ \\
\hline Novel optimization pendulum & 3.21 & 124.14 \\
Before optimization pendulum & 6.24 & 105.47 \\
\hline
\end{tabular}

6.2 Harmonic response analysis

208 In harmonic response analysis, the natural frequency of pendulum structure for a seismometer are analyzed under 209 forced vibration. The amplitude frequency characteristics of novel topology optimization pendulum and before 210 optimization pendulum using harmonic response analysis under ANSYS Workbench are implemented. In simulation 211 experiment, the amplitude of input sine vibrational signals is $10 \mathrm{~N}$, and the frequency is $0-10 \mathrm{~Hz}$ at $0.2 \mathrm{~Hz}$ intervals. The 212 harmonic response measurement results of novel topology optimization pendulum and before optimization pendulum are 213 illustrated in Fig. 7. It can be seen from Fig.7 that the maximum output stress of novel topology optimization pendulum 214 is $77.45 \mathrm{MPa}$ at $3.21 \mathrm{~Hz}$. And the output voltage of before optimization structure pendulum is $13.50 \mathrm{MPa}$ at $6.24 \mathrm{~Hz}$. 215 Compared with before optimization structure pendulum, the natural frequency of novel topology optimization pendulum 216 has reduced by about $48.55 \%$, as well as the maximum output stress of novel topology optimization pendulum has increased 4.7 times. 


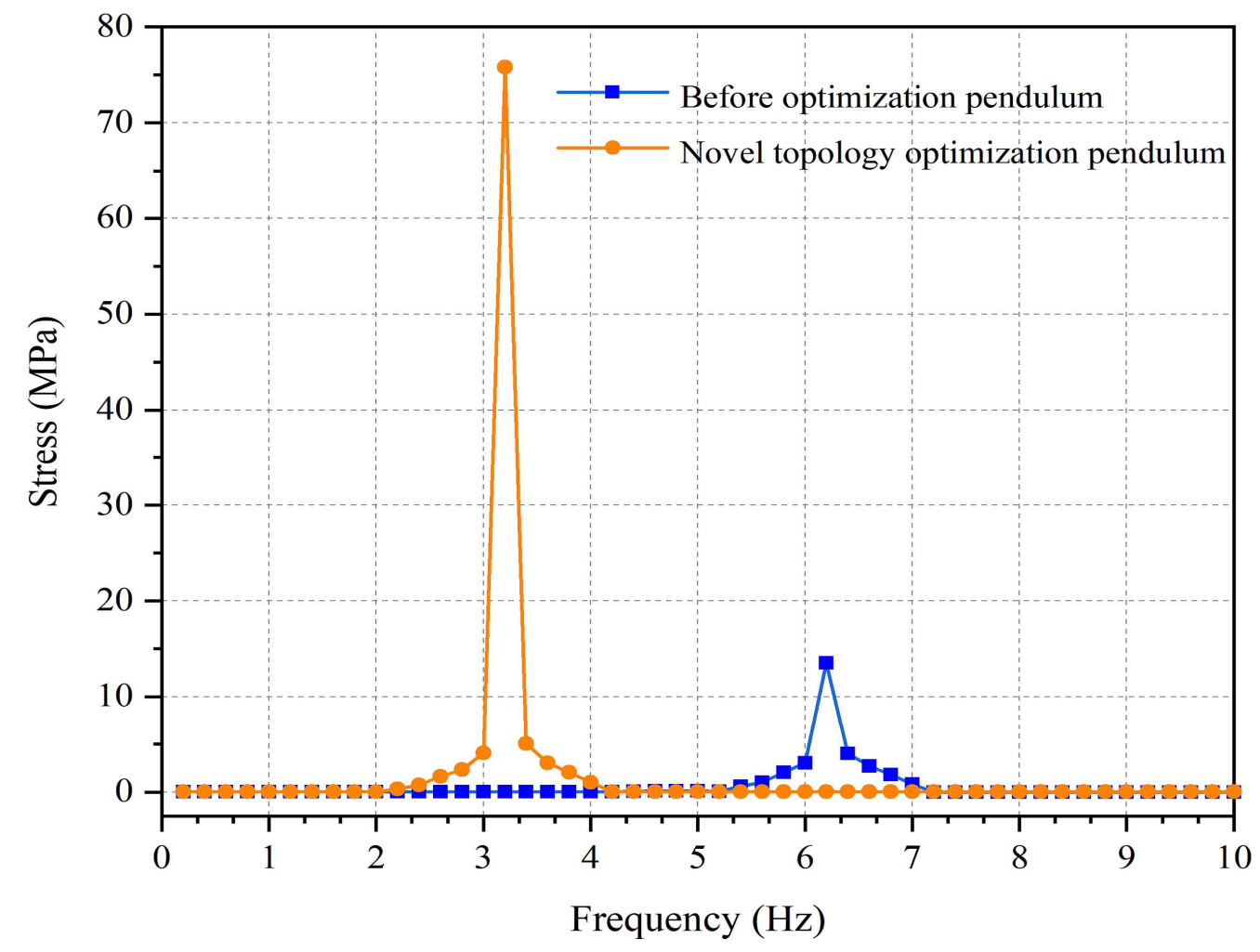

Fig. 7. Harmonic response analysis results of the two kinds of pendulum.

\section{7.Experiments and discussions}

\subsection{Modal testing experiments}

In order to validate theoretical and simulation results, the natural frequency and spurious frequency characteristics of two pendulum structures are measured in Hebei key laboratory of seismic disaster instrument and monitoring technology.

225 Figure 8 shows the material object of novel structure pendulum with topology optimization leaf spring. The modal testing experiments using the Brüel \& Kjær (BK) vibration test system is represented in Fig. 9. The force hammer knocks the pendulum at excitation points sticking accelerometers. After charge amplification, the vibration signal is obtained from the six-channel data collector. The signal modal analysis with BK pulse reflex modal fitting software can be determined at the workstation. 


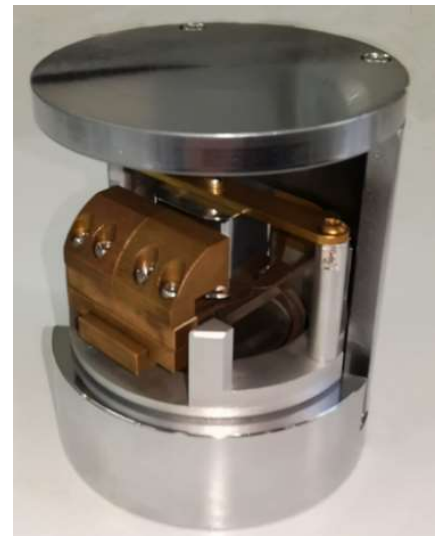

Fig.8. Material object of novel optimization structure pendulum

In the experiments, the 8206-002 series small force hammer is used as the vibration equipment. A BK type 3050-A-060 six-channel data collector, 4508-001 accelerometer and BK pulse labshop test system is adopted as test equipment. The number of excitation points is set to 20 . And the sampling frequency of the data collector is set to $200 \mathrm{~Hz}$

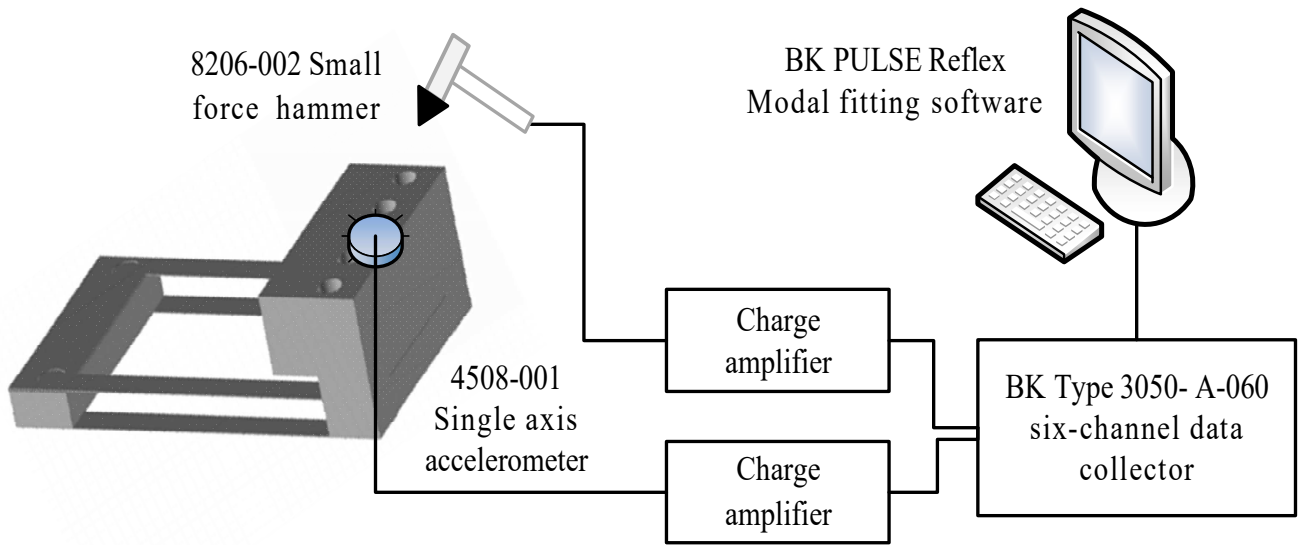

Fig. 9. The hardware set diagram for mode test experiment

The modal testing measurement experimental results of novel topology optimization pendulum and before optimization pendulum are compared in Fig. 10. The blue line is measurement experimental result of before optimization pendulum, and the orange line is measurement experimental result of novel topology optimization pendulum. The experimental results show that the maximum output signal of novel topology optimization pendulum is $3.23 \mathrm{~Hz}$ represented with natural frequency. And the maximum output signal of before optimization pendulum is $6.30 \mathrm{~Hz}$ as its natural frequency. As well as the spurious frequency of novel topology optimization pendulum and before optimization one with the second maximum output signal are $124.83 \mathrm{~Hz}$ and $106.24 \mathrm{~Hz}$, respectively. Compared with before optimization structure pendulum, the natural frequency of novel topology optimization pendulum has reduced by about $48.73 \%$, as well as the spurious frequency has increased by $17.50 \%$. 


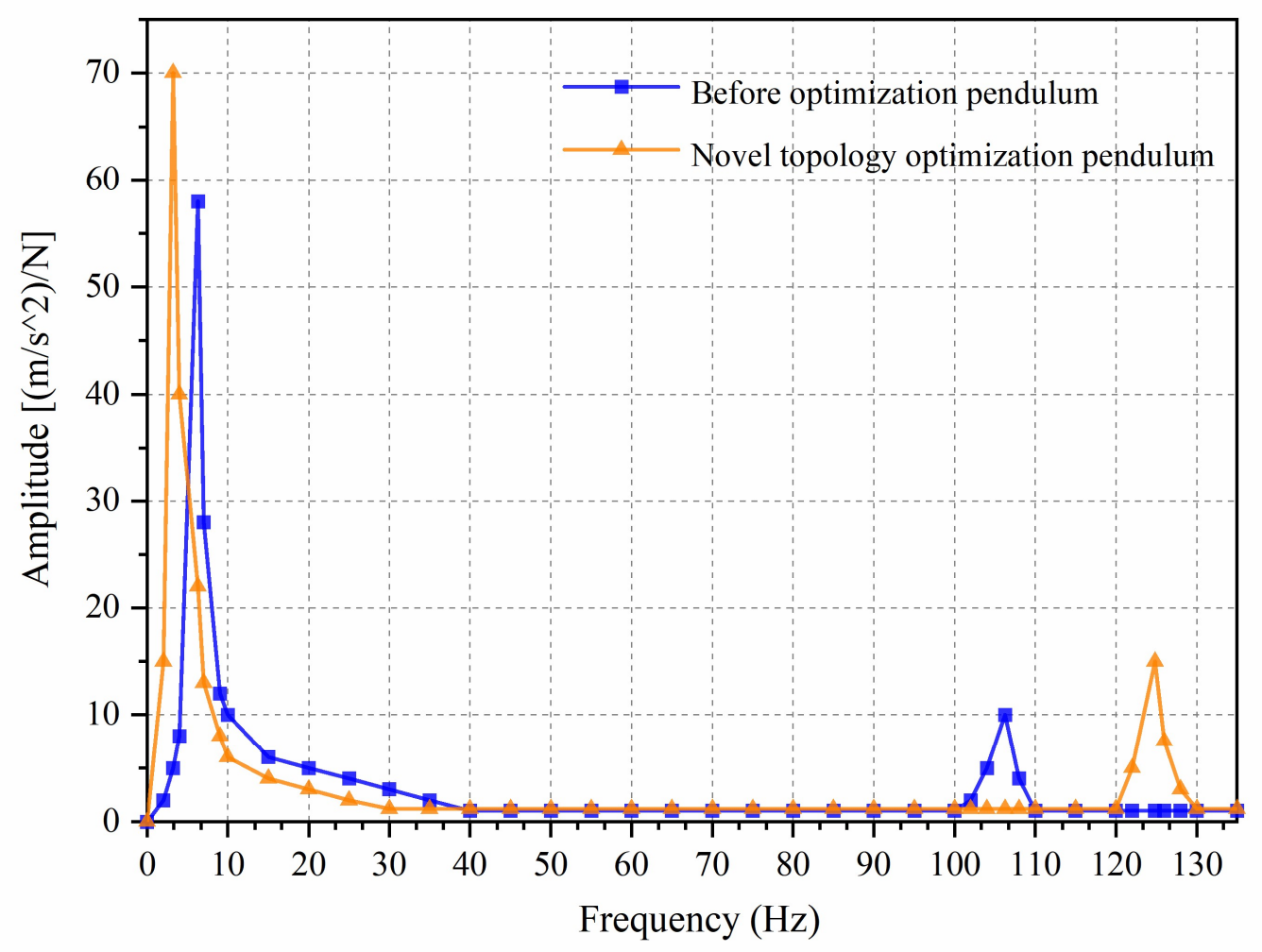

Fig. 10. Frequency response experimental results of two kinds of pendulum

In the meantime, simulation and measurement experimental results of natural frequency and spurious frequency characteristics with two pendulum structures are compared in Table 2. Compared with finite element simulation analysis, the measurement experimental results of natural frequency and spurious frequency characteristics of two pendulum structures has error within $0.96 \%$. This verifies validity of simulation model establishment and frequency characteristic analysis results.

Table 2 Frequency comparison of the novel optimization pendulum and before optimization pendulum.

Frequency value of before optimization pendulum $(\mathrm{Hz})$

Natural frequency Spurious frequency
Frequency value of novel optimization pendulum $(\mathrm{Hz})$

\begin{tabular}{lcccc}
\hline Simulation & 6.24 & 105.47 & 3.21 & 124.14 \\
Experiment & 6.30 & 106.24 & 3.23 & 124.83 \\
Error & $0.96 \%$ & $0.73 \%$ & $0.62 \%$ & $0.56 \%$ \\
\hline
\end{tabular}

\subsection{Shaking table test experiments}

To analyze the effective frequency bandwidth of seismometer influenced by the natural frequency and 

spurious frequency of pendulum, we test the amplitude frequency characteristics of seismometer by shaking table test measurement experiments conducted in Hebei key laboratory of seismic disaster instrument and monitoring technology. In measurement experiments, the seismometer is fixed on the ultra-low frequency standard vibration platform. Its frequency range is $0.0002-160 \mathrm{~Hz}$, the maximum velocity is $0.1 \mathrm{~m} / \mathrm{s}$, and the maximum displacement is $0.15 \mathrm{~m}$. Sinusoidal signals amplified by the power amplifier to drive the standard shaking table, which are measured by the novel optimization seismometer. The amplitude of input sinusoidal signal is $0.5 \mathrm{~cm} / \mathrm{s}$, and its frequency is $0.01-150 \mathrm{~Hz}$.

Figure 11 illustrates the shaking table test measurement experimental results of novel optimization seismometer and before optimization seismometer, the blue line is amplitude frequency characteristics of before optimization seismometer, and the orange line is amplitude frequency characteristics of novel optimization structure seismometer. The horizontal axis is the frequency of input sine vibration signals (in hertz), and the vertical axis is the normalized output amplitude. From Fig.11, it can be seen that the $3 \mathrm{~dB}$ effective frequency bandwidth of novel seismometer is expanded with the topology optimization leaf spring from $1 \mathrm{~s}-51 \mathrm{~Hz}$ to $4 \mathrm{~s}-78 \mathrm{~Hz}$, which is $55.50 \%$ higher than before optimization seismometer. So the effective frequency bandwidth of the novel seismometer with topology optimization leaf spring has improvement. 


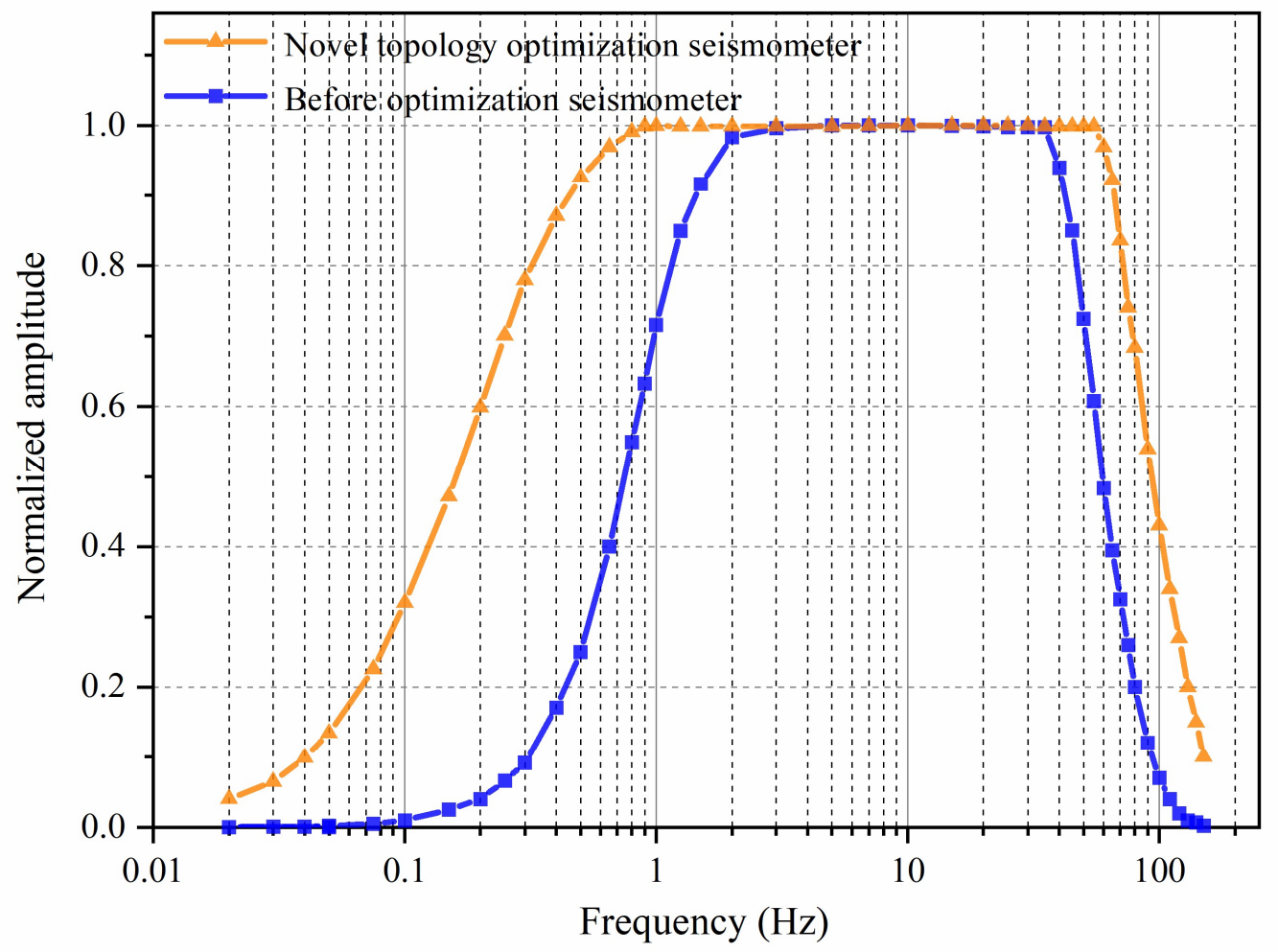

Fig. 11. Vibration measurement experimental results of novel optimization seismometer and before optimization seismometer

\section{Conclusions}

The leaf spring structure is a key factor that affects effective frequency bandwidth performance of the seismometer. This paper has demonstrated that using novel topology optimization structure can expand effective frequency bandwidth of seismometer with lower natural frequency and higher spurious frequency of pendulum. Finite element simulation experiments using ANSYS Workbench show that, compared with before optimization structure pendulum, the natural frequency of novel topology optimization pendulum has reduced by about $48.55 \%$, as well as the spurious frequency of novel topology optimization pendulum has increased $17.70 \%$. The real vibration measurement experimental results indict that the frequency bandwidth of topology optimization seismometer is improved to $[4 \mathrm{~s}, 78 \mathrm{~Hz}]$ with an increase of $55.50 \%$ over before optimization seismometer frequency bandwidth of $[1 \mathrm{~s}, 51 \mathrm{~Hz}]$. In the future, the sensitivity of a seismometer using the proposed novel topology optimization structure will be analyzed.

\section{CRediT authorship contribution statement}


Li: Software, Investigation, Validation.

\section{Declaration of Competing Interest}

The authors declare that they have no known competing financial interests or personal relationships that could have appeared to influence the work reported in this paper.

\section{Acknowledgments}

This work is supported by the Special Fund of Fundamental Scientific Research Business Expense for Higher School of Central Government (Projects for creation teams) (Grant No. ZY20215101), and the National Natural Science Foundation of China (Grant No. 60475028). The research work of this paper is performed at Hebei Key Laboratory of Seismic Disaster Instrument and Monitoring Technology.

\section{References}

[1] G.L Li, J.B Wang, D.Y Chen, et al., An Electrochemical, Low-frequency seismic micro-sensor based on MEMS with a force-balanced feedback system, Sensors. 17 (9) (2017) 2103, http://dx.doi.org/10.3390/s17092103.

[2] J. Lin, H. Gao, X.F. Wang, et al., Effect of temperature on the performance of electrochemical seismic sensor and the compensation method, Measurement. 155 (2020) 107518, http://dx.doi.org/10.1016/j.measurement.2020.107518.

[3] J.Y. Wang, B.X. Hu, W. Li, et al., Design and application of fiber Bragg grating (FBG) geophone for higher sensitivity and wider frequency range, Measurement. $79 \quad$ (2016) 228-235, 307 http://dx.doi.org/10.1016/j.measurement.2015.09.041.

308 [4] X.L. Zhang, X.M. Liu, F.X. Zhang, et al., Reliable high sensitivity FBG geophone for low frequency seismic acquisition, Measurement. 129 (2018) 62-67, http://dx.doi.org/10.1016/j.measurement.2018.07.009.

310 [5] Y.T Teng, X.X Hua, H.Y Lu, Research on magnetoelectric seismometer's low frequency extension technology, Appl. 311 Mech. Mater. 664 (2014) 268-273, http://dx.doi.org/10.4028/www.scientific.net/AMM.664.268.

312 [6] K. Faber, P.W. Maxwell, Geophone spurious frequency: What is it and how does it affect seismic data quality, Can. J.

313 Explor. Geophys. 33 (1-2) (1997) 46-54, http://dx.doi.org/10.1190/1.1826773.

314 [7] D.M. Woo, Geophone spring, U.S. patent 5,134,594, (1992).

315 [8] E. Wielandt, G. Streckeisen, The leaf-spring seismometer: Design and performance, Bull. Seismol. Soc. Am. 72 (6) $316 \quad$ (1982) 2349-2367.

317 [9] D.P. Yang, N.R. Li, C.Y. Liu, et al., Note: Improving the performance of a geophone through suspension system configuration, Rev. Sci. Instrum. 85 (12) (2014) 84-92, http://dx.doi.org/10.1063/1.4897183. 
319 [10] Y. Xin, H.S. Sun, C. Guo, et al., Note: A novel optimization cantilever beam for low frequency high performance 320 piezoelectric geophone, Rev. Sci. Instrum. 88 (6) (2017) 066105-1-066105-3, http://dx.doi.org/10.1063/1.4986810.

321 [11] Z.J. Yao, Y.L. Duan, L.L. Li, et al., Bandwidth extension of seismometer by using a novel topology leaf spring, Rev. 322 Sci. Instrum. 90 (7) (2019) 076109-1-076109-3, http://dx.doi.org/10.1063/1.5097026.

323 [12] I. Muramatsu, T. Sasatani, I. Yokoi, Velocity-type strong-motion seismometer using a coupled pendulum: Design 324 and Performance, Bull. Seismol. Soc. Am. 91 (3) (2001) 604-616, http://dx.doi.org/10.1785/0120000023.

325 [13] O. Sigmund, K. Maute, Topology optimization approaches, Struct. Multidisc. Optim. 48 (6) (2013) 1031-1055, 326 http://dx.doi.org/10.1007/s00158-013-0978-6.

327 [14] H.A. Eschenauer, N. Olhoff, Topology optimization of continuum structures: A review, Appl. Mech. Rev. 54 (4) 328 (2001) 331-389, http://dx.doi.org/10.1115/1.1388075.

329 [15] W.J. Zuo, K. Saitou, Multi-material topology optimization using ordered SIMP interpolation, Struct. Multidisc. 330 Optim. 55 (2) (2016) 477-491, http://dx.doi.org/10.1007/s00158-016-1513-3.

331 [16] W.M. Vicente, Z.H. Zuo, R. Pavanello, et al., Concurrent topology optimization for minimizing frequency responses 332 of two-level hierarchical structures, Comput. Methods Appl. Mech. Engrg. 301 (2016) 116-136, 333 http://dx.doi.org/10.1016/j.cma.2015.12.012.

334 [17] Z.D. Li, X.J. Liu, Guide-weight method on solving topology optimization problems under single load case, J. Mech. 335 Eng. 47 (15) (2011) 107-114, http://dx.doi.org/10.3901/JME.2011.15.107.

336 [18] K. Svanberg, The method of moving asymptotes - a new method for structural optimization, Int. J. Numer. Meth. 337 Eng. 24 (2) (1987) 359-373, http://dx.doi.org/10.1002/nme.1620240207.

338 [19] K. Svanberg, A class of globally convergent optimization methods based on conservative convex separable 339 approximations, SIAM J. Optimiz. 12 (2) (2002) 555-573, http://dx.doi.org/10.1137/S1052623499362822.

340 [20] Y. Okamoto, R. Hoshino, S. Wakao, et al., Improvement of torque characteristics for a synchronous reluctance 341 motor using MMA-based topology optimization method, IEEE Trans. Magn. 54 (3) (2018) 1-4, 342 http://dx.doi.org/10.1109/TMAG.2017.2762000. 